\title{
Percutaneous Endoscopic Gastrostomy Tube Is a Negative Prognostic Factor for Recurrent/ Metastatic Head and Neck Cancer
}

\author{
MARCO SIANO $^{1}$, NADINE JARISCH ${ }^{2}$, MARKUS JOERGER $^{1}$ and VITTORIA ESPELI ${ }^{3}$ \\ ${ }^{1}$ Cantonal Hospital St. Gallen, Oncology/Hematology Clinic, St. Gallen, Switzerland; \\ ${ }^{2}$ Cantonal Hospital St. Gallen, Department of Internal Medicine, Nutrition Counseling, St. Gallen, Switzerland; \\ ${ }^{3}$ Oncology Insitute of Southern Switzerland, Ospedale San Giovanni, Bellinzona, Switzerland
}

\begin{abstract}
Background/Aim: Recurrent/metastatic head and neck squamous cell cancer ( $r / m H N S C C$ ) patients often need a percutaneous endoscopic gastrostomy feeding tube (PEG). Among known prognostic factors, PEG could be prognostic as well. Patients and Methods: We retrospectively analyzed r/mHNSCC patients referred for systemic treatment. Kaplan-Meier and multivariate cox regression models were applied to assess prognostic impact of PEG. Results: One hunderd and ten patients were identified, 42 had a PEG at treatment start. Median survival from start of 1 st-line systemic treatment was 8 months (95\%CI=6.5-12.0 months), 4.5 months (95\%CI=2.5-7.0 months) for patients with PEG and 11.5 months (95\%CI=7.5-14.5 months) without PEG (adjusted $H R=1.98, p=0.011$ ). Similarly, survival from first recurrence of distant metastases was lower in patients with PEG as compared to patients without (7.5 vs. 15.5 months, adjusted HR=2.60, $p<0.001)$. Conclusion: Presence of PEG feeding tube has an unfavourable prognostic impact on survival in patients with $\mathrm{r} / \mathrm{mHNSCC}$. While any causality remains speculative, potential complications should be appreciated before PEG implantation.
\end{abstract}

HNSCC includes a variety of subtypes depending on the organ of origin where cancer develops. The incidence depending on primary site and sub-type is around $5-10 / 100,000$ being the 5 th most common cancer worldwide (1). The most common

This study was reported in abstract form at the Annual Meeting of the European Society of Medical Oncology ESMO 2016.

Correspondence to: Marco Siano,Cantonal Hospital St. Gallen, Oncology/Hematology Clinic, 9000 St. Gallen, Switzerland. Tel: +41 714943539, Fax:+41 714946325, e-mail: marco.siano@kssg.ch

Key Words: Percutaneous endoscopic gastrostomy, head and neck cancer, prognostic, clinical. cause of these cancers remains smoking (2) and alcohol abuse, despite the upraising of HPV-related cancers notably in western countries (3). The latter show, independently of the applied treatment modality throughout, a better prognosis and comprise mostly oropharyngeal cancers and to lower extent other head and neck cancer subtypes (4). HNSCC's are cured with surgery and/or radio-chemo-therapy in about two thirds of cases. Nevertheless, in locally advanced tumor stages III and IV, local recurrences or distant metastases occur in about $40-60 \%$ of patients $(5,6)$.

Patients with HNSCC frequently require percutaneous endoscopic gastrostomy (PEG) feeding tubes during the course of their disease. In the recurrent and/or metastatic setting, therapeutic PEG is supposed to compensate for impaired swallowing and aspiration, and to improve patients' nutrition status with a potential positive effect on outcome (7). Even if there might be issues with the handling of PEG feeding tubes, patients in general perceive them as beneficial (8).

Either placed prophylactically, before curatively-intended local treatment, or therapeutically in the course of treatment, patients usually get rid of their PEG feeding tube after completion of treatment and resolution of adverse events. However, sometimes PEG removal is not warranted due to organ dysfunction after radiotherapy, rapid tumor recurrence, persistence or progressive metastatic disease, with related symptoms (dysphagia, dysphonia, strictures). Also, in case of tumor recurrence, depending on the location and extend of disease and related symptoms, PEG feeding tube placement may become necessary, particularly in cases of impending tumor stenosis and related complications.

While the presence of PEG feeding tubes is studied extensively in locally advanced disease, this is not the case for $\mathrm{r} / \mathrm{mHNSCC}$ (9). Furthermore, no data have been generated investigating a potential prognostic impact of PEG feeding tubes in this setting. The aim of this analysis was to assess whether the presence of a PEG feeding tube is an independent prognostic factor in $\mathrm{r} / \mathrm{mHNSCC}$. 


\section{Patients and Methods}

We retrospectively analyzed patients with $\mathrm{r} / \mathrm{mHNSCC}$ referred for palliative systemic treatment between 2000 and 2014. Patients disease, treatment characteristics and outcome were assessed, including presence of PEG at the start of systemic therapy. Known prognostic factors according to Argiris et al (Performance status, tumor cell differentiation, site of primary tumor, prior radiation, response to chemotherapy) were assessed and considered for analysis (10).

Statistics. The primary objective of the study was to assess the quantitative prognostic impact of a PEG feeding tube in this group of patients. The prognostic impact of the presence of a PEG feeding tube on survival from the time of first occurrence of distant metastases and start of palliative 1st-line systemic treatment was calculated using Kaplan-Meier survival analysis and multivariate Cox regression models. Known prognostic factors including patient age, gender, ECOG performance score, histological grading were used as co-variates and included in multivariate Cox proportional hazard models (10). The study had a power of $70 \%$ to detect a $50 \%$ deterioration of OS (corresponding to a hazard ratio of 2.0) with a type-I error of $5 \%$. All tests of significance were two-sided; $p<0.05$ was considered significant. Statistical analyses were performed using STATA 11.0 (STATA Corp, College Station, Texas, USA). The local Ethics Committee approved data collection and analysis.

\section{Results}

Patient characteristics. We included 110 patients with mHNSCC evaluable for analysis between January 2000 and May 2014. Almost all patients had metastatic state at referral $(84 \%)$, whereas $16 \%$ had recurrence without distant disease. 100 patients received first-line therapy. Forty-two patients had a PEG a time of palliative 1st-line systemic treatment. Patient characteristics are further elucidated in Table I.

Survival and presence of PEG. Median survival from the start of 1 st line systemic treatment was 8.0 months $(95 \%$ $\mathrm{CI}=6.5-12.0$ months). ECOG PS was the strongest prognostic factor in our cohort $(\mathrm{HR}=2.33, p<0.001)$. Overall survival from the time of 1st-line palliative systemic treatment was 4.5 months (95\% CI, 2.5-7.0 months) for patients carrying a PEG and 11.5 months (95\% CI, 7.5-14.5 months) for patients without PEG (adjusted HR=1.98, $p=0.011$ ) (Figure 1). Survival from first occurrence of distant metastases was significantly lower in PEG carriers as compared to patients without a PEG (7.5 v 15.5 months, adjusted HR=2.60, $p<0.001$ ) (Figure 2). Multivariate analyses for prognostic factors in relation to survival are shown in Table II.

\section{Discussion}

Our retrospective analysis of patients with $\mathrm{r} / \mathrm{mHNSCC}$ shows that presence of PEG feeding tube is an independent negative prognostic factor for survival and should be
Table I. Patient demographics.

\begin{tabular}{|c|c|c|c|}
\hline \multirow[b]{2}{*}{$\mathrm{n}=110$} & \multicolumn{2}{|c|}{$\mathrm{n}(\%)$} & \multirow[t]{2}{*}{$p$-Value } \\
\hline & $\begin{array}{c}\text { with PEG } \\
42\end{array}$ & $\begin{array}{c}\text { without PEG } \\
68\end{array}$ & \\
\hline $\begin{array}{l}\text { Median age } \\
\text { Range }\end{array}$ & 59 & 63 & 000 \\
\hline \multicolumn{4}{|l|}{ Gender } \\
\hline Male & $36(33)$ & $57(52)$ & \multirow[t]{2}{*}{0.79} \\
\hline Female & $6(5)$ & $11(10)$ & \\
\hline \multicolumn{4}{|l|}{ ECOG PFS } \\
\hline 0 & $13(12)$ & $17(16)$ & \multirow[t]{3}{*}{0.19} \\
\hline 1 & $17(15)$ & $41(37)$ & \\
\hline$>2$ & $12(11)$ & $10(9)$ & \\
\hline \multicolumn{4}{|c|}{ Prior local radiotherapy } \\
\hline Yes & $38(35)$ & $52(47)$ & \multirow[t]{2}{*}{0.06} \\
\hline No & $4(4)$ & $16(14)$ & \\
\hline \multicolumn{4}{|l|}{ Stage at diagnosis } \\
\hline I & - & $3(3)$ & \multirow[t]{4}{*}{0.52} \\
\hline II & $3(3)$ & $4(4)$ & \\
\hline III & $6(5)$ & $7(6)$ & \\
\hline IV & $33(30)$ & $54(49)$ & \\
\hline \multicolumn{4}{|l|}{ Primary tumor site } \\
\hline Oropharynx & $14(13)$ & $18(16)$ & \multirow[t]{5}{*}{0.49} \\
\hline Hypopharynx & $4(4)$ & $14(13)$ & \\
\hline Larynx & $10(9)$ & $12(11)$ & \\
\hline Oral Cavity & $13(12)$ & $16(15)$ & \\
\hline Other & $1(1)$ & $8(7)$ & \\
\hline \multicolumn{4}{|l|}{ Stage at recurrence } \\
\hline Locally advanced & $9(8)$ & $8(7)$ & \multirow[t]{2}{*}{0.17} \\
\hline Metastatic & $33(30)$ & $60(55)$ & \\
\hline \multicolumn{4}{|l|}{ Grading WHO } \\
\hline $0-1$ & $6(5)$ & $5(5)$ & \multirow[t]{3}{*}{0.11} \\
\hline 2 & $23(21)$ & $42(38)$ & \\
\hline 3 & $13(12)$ & $21(19)$ & \\
\hline
\end{tabular}

considered with the already established ones and published by other groups (10).

Other co-factors, not described so far, could also be important and interplay with our primary observation, leading to the placement or retention of a PEG feeding tube: anatomical localization of persistent or recurrent disease, pre-existent cancer cachexia or altered nutritional status, functional restrictions caused by acute or late treatment related toxicity (radiation mucositis, impaired swallowing, aspiration). Therefore, even if any direct causality of PEG with survival remains speculative, the simple fact that its presence is prognostic is important information for the clinician. Prognostic value of PEG feeding tube was already described for radiotherapy in HNSCC patients but not so far for $\mathrm{r} / \mathrm{mHNSCC}$, making our analysis pivotal (9).

A major limitation of our analysis was its retrospective nature, not ruling out some important bias, even though the analyzed groups were well balanced. Advantages 


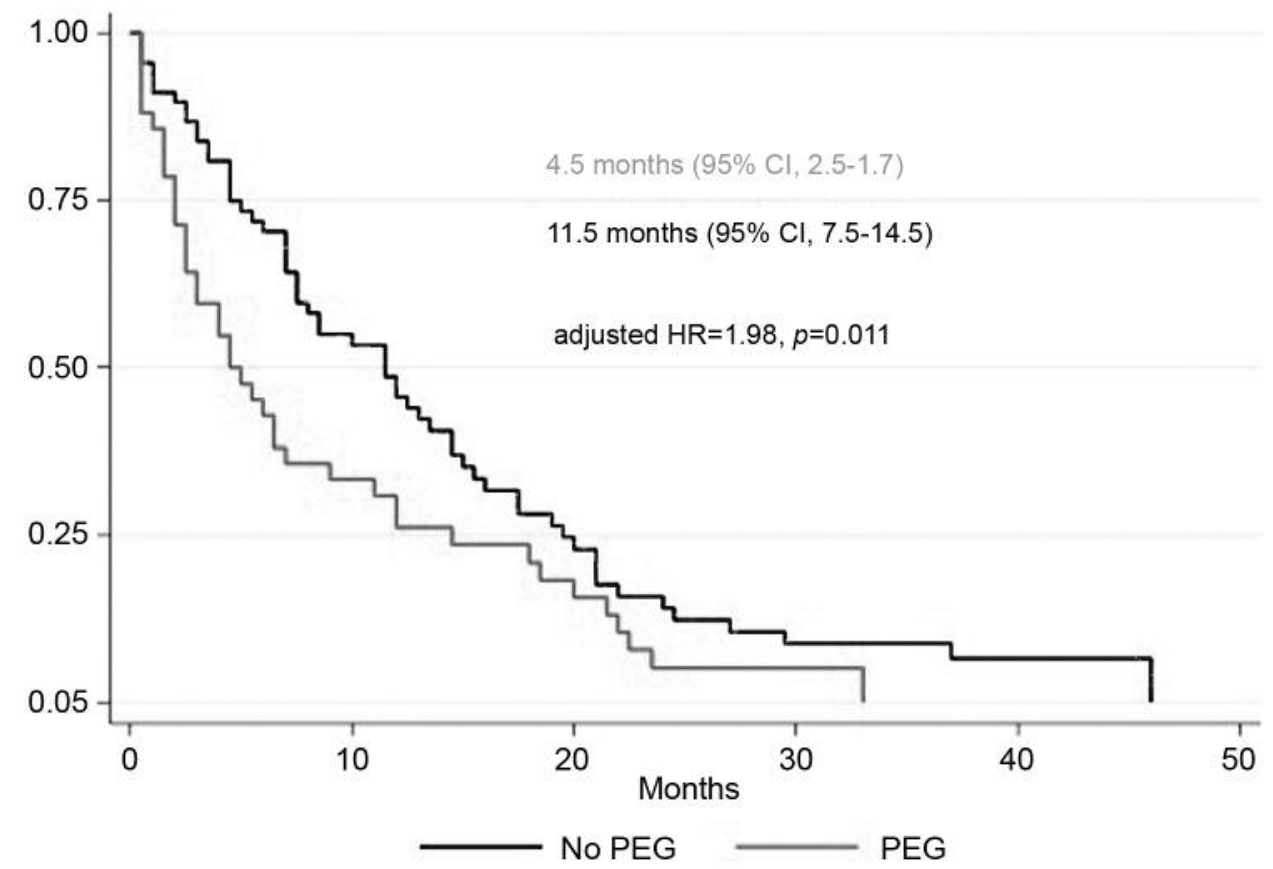

Figure 1. Overall survival from the time of 1st-line palliative systemic treatment. The Kaplan-Meier survival curve of patients harboring a PEG in red versus patients without PEG in blue from start of first-line systemic treatment is shown.

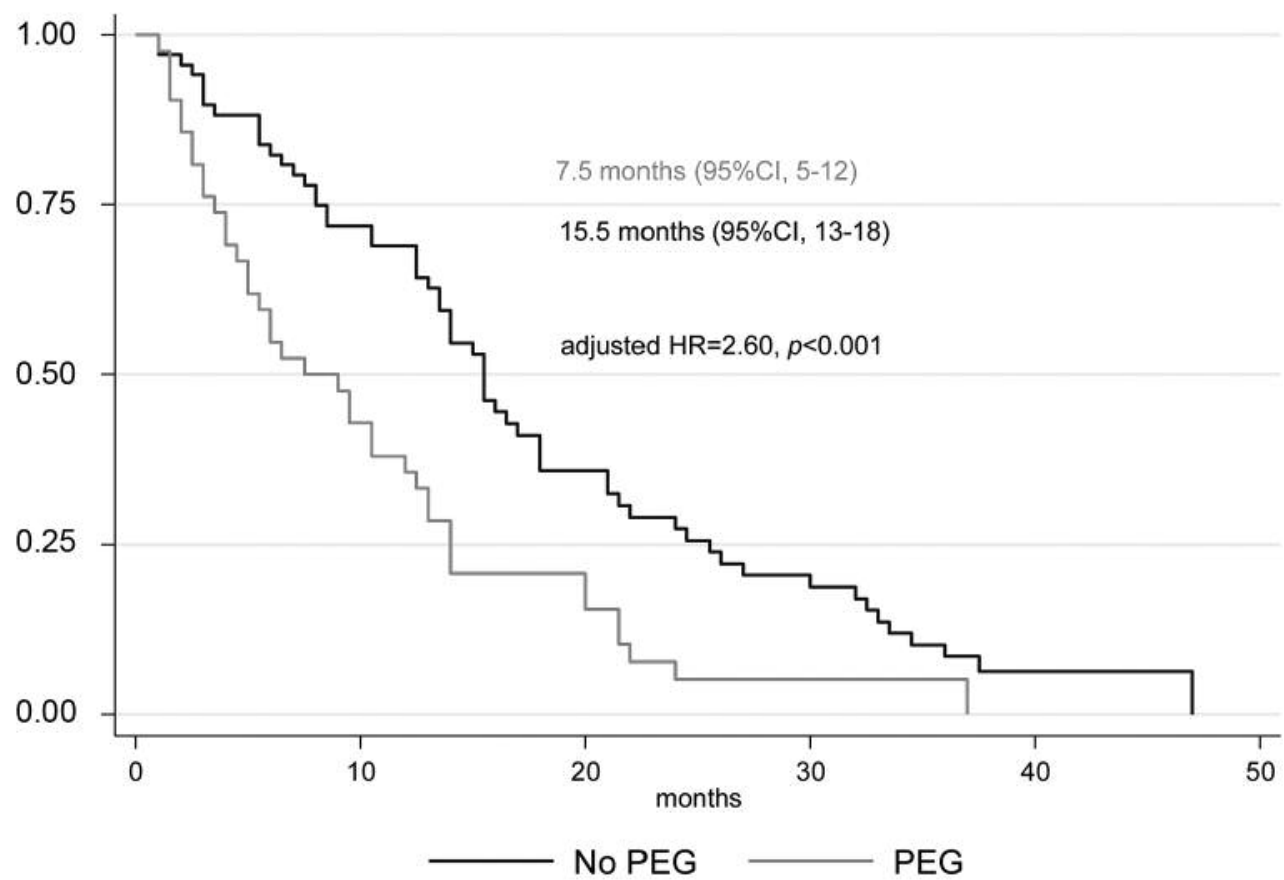

Figure 2. Survival from first occurrence of distant metastases. The Kaplan-Meier survival curve of patients harboring a PEG in red versus patients without PEG in blue from advent of recurrence or metastatic disease is shown. 
Table II. Multivariate analyses for prognostic factors related to survival from start of 1st-line treatment (A) presence of distant metastases $(B)$.

A

\begin{tabular}{lccc}
\hline Risk factors & Hazard ratios & $95 \% \mathrm{CI}$ & $p$-Value \\
\hline Presence of PEG & 1.98 & $1.17-3.35$ & 0.011 \\
Age & 0.78 & $0.34-1.77$ & 0.551 \\
Gender & 0.75 & $0.39-1.43$ & 0.386 \\
ECOG PS & 2.34 & $1.65-3.31$ & 0.000 \\
Tumor grading & 0.80 & $0.58-1.11$ & 0.186 \\
Local radiotherapy & 0.76 & $0.40-1.43$ & 0.389 \\
\hline
\end{tabular}

B

\begin{tabular}{lccc}
\hline Risk factors & Hazard ratios & $95 \% \mathrm{CI}$ & $p$-Value \\
\hline Presence of PEG & 2.60 & $1.53-4.43$ & 0.001 \\
Age & 1.46 & $0.62-3.42$ & 0.383 \\
Gender & 0.94 & $0.49-1.81$ & 0.861 \\
ECOG PS & 1.85 & $1.33-2.58$ & 0.000 \\
Tumor grading & 0.70 & $0.50-0.98$ & 0.037 \\
Local radiotherapy & 0.90 & $0.47-1.25$ & 0.74 \\
\hline
\end{tabular}

retrieved for the patients by their PEG should be balanced against potential complications and disadvantages like infections and peritonitis, caused by displacement and systemic treatments (11). For instance, classic hematotoxic chemotherapy with subsequent neutropenia or anti-epidermal growth factor receptor antibodies with related cutaneous rash or wound healing can be made accountable for. Instruction for handling, maintenance and nutrition intake, so as compliance by the patients themselves are also of major importance to retain advantages and avoid complications of PEG tube. Head and neck cancer patients comprise a special patient category often characterized by a noxious lifestyle not always showing best compliance (12).

Other prognostic factors are also worth to be mentioned besides the ones already established by Argiris et al for $\mathrm{r} / \mathrm{mHNSCC}$ and relevant for our analysis (10). Primarily loss of weight, which is related to decreased survival as shown in a recent prospective study, in which a weight loss grading system was developed showing a relationship between weight change and survival (13). In this publication, the described grades showed good survival discrimination for metastatic cancers where median survival significantly differed from 12.2 months for grade 0 to 3.8 months for grade $4(p<0.001)$. For head and neck cancer of any stage, median survival significantly differed from 77.9 months for grade 0 to 6.1 months for grade 4 ( $p=0.001)$.

Potential complications should be considered before PEG implantation and knowledge of worse prognosis in these patients helps decision taking during further treatment. More research should be undertaken to investigate the indication and clinical benefit of PEG tube placement. Currently established prognostic factors could be insufficient for adequate adjusted multivariate analysis in $\mathrm{r} / \mathrm{mHNSCC}$. If other and larger patient cohorts confirm our observation, PEG presence should be considered as prognostic factor in future clinical trials.

\section{Conclusion}

In summary, in a patient cohort of patients with $\mathrm{r} / \mathrm{mHNSCC}$, we observed the prognostic value of presence of a PEG feeding tube in disregard of the primary reason for placement or tenure, suggesting to be an independent prognostic factor for these patients.

\section{Funding}

The work was performed and the Cantonal Hospital St. Gallen Switzerland without receiving specific financial support.

\section{Conflicts of Interest}

There are no potential conflicts of interest to be reported.

\section{References}

1 Jemal A, Bray F, Center MM, Ferlay J, Ward E and Forman D: Global cancer statistics. CA Cancer J Clin 6: 69-90, 2011.

2 Krayzler E and Nagler RM: Cigarette smoke-induced effects on the cell cycle in oral cancer cells: Reduction of $\mathrm{G}_{2} / \mathrm{M}$ fraction. Cancer Genomics Proteomics 12: 73-76, 2015.

3 Leemans CR, Braakhuis B and Brakenhoff R: Molecular biology of head and neck cancer. Nat Rev Cancer 11: 9-22, 2011.

4 Ang KK, Harris J, Wheeler R, Weber R, Rosenthal DI, NguyenTân PF, Westra WH, Chung CH, Jordan RC, Lu C, Kim H, Axelrod R, Silverman CC, Redmond KP and Gillison ML: Human Papillomavirus and Survival of Patients with Oropharyngeal Cancer. N Engl J Med 363: 24-35, 2010.

5 Piccirillo JF, Costas I, Riechmann ME: Cancers of the Head and Neck, SEER Survival Monograph: Cancer Survival Among Adults: U.S. SEER Program, 1988-2001, Patient and Tumor Characteristics. Ries LAG, Young JL, Keel GE, Eisner MP, Lin YD, Horner M-J. National Cancer Institute Bethesda: 7-22, 2007.

6 Noguti J, De Moura CF, De Jesus GP, Da Silva VH, Hossaka TA, Oshima CT and Ribeiro DA: Metastasis from oral cancer: an overview. Cancer Genomics Proteomics 9: 329-335, 2012.

7 Brown T, Banks M, Hughes BGM, Lin C, Kenny LM and Bauer JD: Impact of early prophylactic feeding on long term tube dependency outcomes in patients with head and neck cancer. Oral Oncol 72: 140-149, 2017.

8 Kwong JP, Stokes EJ, Posluns EC, Fitch MI, McAndrew A and Vandenbussche KA: The experiences of patients with advanced head and neck cancer with a percutaneous gastrostomy tube: A qualitative descriptive study. Nutr Clin Pract 29: 526-533, 2014. 
9 Shaw SM, Flowers H, O'Sullivan B, Hope A, Liu LW and Martino R: The effect of prophylactic percutaneous endoscopic gastrostomy (PEG) tube placement on swallowing and swallowrelated outcomes in patients undergoing radiotherapy for head and neck cancer: a systematic review. Dysphagia 30: 152-175, 2015.

10 Argiris A, Li Y, Forastiere A: Prognostic factors and long-term survivorship in patients with recurrent or metastatic carcinoma of the head and neck. Cancer 101: 2222-2229, 2004.

11 Olsen R, Karam I, Wilson G, Bowman A, Lee C and Wong F: Population-based comparison of two feeding tube approaches for head and neck cancer patients receiving concurrent systemicradiation therapy: is a prophylactic feeding tube approach harmful or helpful? Support Care Cancer 21: 3433-3439, 2013.
12 Ostroff JS, Hay JL, Schantz SP and Maher MM: A survey of smoking status and cancer risk perceptions among participants attending a hospital-based head and neck screening program. Psychol Health 14: 979-990, 2000.

13 Martin L, Senesse P, Gioulbasanis I, Antoun S, Bozzetti S, Deans C, Strasser F, Thoresen L, Jagoe RT, Chasen M, Lundholm K, Bosaeus I, Fearon $\mathrm{KH}$ and Baracos VE: Diagnostic criteria for the classification of cancer-associated weight loss. J Clin Oncol 33: 90-99, 2015.

Received April 5, 2018

Revised May 11, 2018

Accepted May 15, 2018 